\title{
Thermohaline mixing in super-AGB stars
}

\author{
L. Siess ${ }^{1,2}$ \\ 1 Institut d'Astronomie et d'Astrophysique, Université Libre de Bruxelles (ULB), CP 226, 1050 Brussels, Belgium \\ e-mail: siess@astro.ulb.ac.be \\ 2 Centre for Stellar and Planetary Astrophysics, School of Mathematical Sciences, Monash University, Victoria 3800, Australia
}

Received 17 November 2008 / Accepted 26 January 2009

\section{ABSTRACT}

\begin{abstract}
Aims. We present the first study of the effects of thermohaline mixing on the structure and evolution of solar-composition super-AGB (SAGB) stars in the mass range $9-11 M_{\odot}$

Methods. We developed and analyzed stellar models taking into account thermohaline mixing and varying mixing efficiencies. Results. In SAGB stars, thermohaline mixing becomes important after carbon has been ignited off-center and it affects significantly the propagation of the flame. In the radiative layers located below the convective carbon-burning zone, a molecular weight inversion is created which allows the efficient transport of chemicals. The outward diffusion of ${ }^{12} \mathrm{C}$ from the CO-rich core into the flame, depletes the burning front of fuel and causes the extinction of the flame before it reaches the center. As a consequence the amount of unburnt carbon can be as high as $2-5 \%$ in mass at the center of the star. During the subsequent thermally pulsing SAGB phase, the high temperature at the base of the convective envelope prevents the development of thermohaline instabilities associated with ${ }^{3} \mathrm{He}$ burning as found in low-mass red giant stars.

Conclusions. In contrast to the case of low-mass RGB stars, thermohaline mixing is unable to alter the surface composition of SAGB stars. We also emphasize that if the SAGB star evolves into an electron-capture supernovae, the ${ }^{12} \mathrm{C}$ remaining in the core may alter the hydrodynamical explosion and modify the explosive nucleosynthesis.
\end{abstract}

Key words. stars: evolution - stars: supernovae: general - nuclear reactions, nucleosynthesis, abundances - instabilities

\section{Introduction}

Thermohaline instabilities are also referred to as double diffusive instabilities and are driven by the fact that the stabilizing component (heat) diffuses more rapidly than the destabilizing one (chemicals). The classical picture consists of a layer of warm, salty water positioned on top of pure cold water. Since heat diffuses faster than salt, a fluid element that is displaced downward remains heavier than its surrounding and continues to sink despite the stabilizing temperature gradient. Mixing proceeds until the two effects compensate each other and the element becomes turbulent. These instabilities produces finger-like patterns and are responsible for the efficient mixing. In the stellar case, the mean molecular weight $(\mu)$ plays the role of the salinity, and the instability develops inside the radiative layers when $\nabla \mu=\mathrm{d} \ln \mu / \mathrm{d} \ln P<0$.

As the extensive literature attests, the effects of thermohaline mixing on the structure and composition of stars have been analyzed in various astrophysical contexts. In binary systems, thermohaline instabilities may be induced when heavy material from the primary star is transferred to its companion. This includes both close and wide low-mass binaries (e.g., Marks \& Sarna 1998; Bitzaraki et al. 2004; Stancliffe et al. 2008), as well as massive systems (e.g., de Grève \& Cugier 1989; Wellstein et al. 2001). However, these instabilities can also develop in single stars as the result of the accretion of metal-rich matter during the phase of planetary formation (e.g. Vauclair 2004) or because of nuclear burning. In this latter case it was realized that thermohaline mixing is likely to operate during the core helium flash in low-mass stars (Thomas 1967) or in the region of $\mathrm{H}$ burning where the ${ }^{3} \mathrm{He}\left({ }^{3} \mathrm{He}, 2 \mathrm{p}\right)^{4} \mathrm{He}$ reactions are active (Ulrich 1972). Using 3D hydrodynamical simulations, Eggleton et al. (2006) detected the presence of this instability at the convective envelope base of a low-mass red giant. This hydrodynamical study was then complemented by stellar evolution computations (Charbonnel \& Zahn 2007; Eggleton et al. 2008), where it was shown that the mean molecular weight inversion created below the convective envelope by the ${ }^{3} \mathrm{He}+{ }^{3} \mathrm{He}$ reactions produces an efficient transport of chemicals. This additional mixing process is activated when the $\mathrm{H}$ burning shell crosses the discontinuity left by the passage of the convective envelope during the first dredge-up, i.e. at the luminosity bump, and accounts for the reduction in the surface abundance of ${ }^{3} \mathrm{He}$, ${ }^{7} \mathrm{Li}$, as well as the ${ }^{12} \mathrm{C} /{ }^{13} \mathrm{C}$ ratio, in agreement with observations of globular cluster stars.

The non-explosive evolution of solar metallicity SAGB stars was first investigated in details by Garcia-Berro and collaborators (Garcia-Berro et al. 1997; Iben et al. 1997; Ritossa et al. 1996, 1999) with special attention to the propagation of a laminar flame after the off-center ignition of carbon. More recently SAGB stars in binary systems have been studied (e.g., Gil-Pons \& Garcia-Berro 2002), non-solar models have been computed (e.g., Gil-Pons et al. 2005; Siess 2007) and the effect of overshooting and numerics have been investigated (e.g., Gil-Pons et al. 2007; Siess 2007; Poelarends et al. 2008).

In the present paper, we explore in more details the effects of thermohaline mixing in SAGB stars, where the off-center ignition of carbon provides favorable conditions for the development of this instability. In Sect. 2, we briefly review the main physical assumptions used in the computations, before discussing in detail the effects of this process on the structure and evolution of SAGB stars. Our analysis proceeds linearly along an 
evolutionary sequence from the main sequence to the thermally pulsing SAGB (TP-SAGB) phase. In Sect. 4, we investigate the effects of varying the mixing efficiency, and in Sect. 5, we analyze how this process operates in stars of various initial stellar masses. A summary is presented in Sect. 6.

\section{Input physics}

The models are computed with version v3.00 of starevol as described by Siess (2007) and Siess \& Arnould (2008). The Schwarzschild criterion is used to delineate the convective boundaries, core overshooting is not accounted for, and the chemical composition is scaled to solar values according to the Grevesse et al. (1996) mixture. The condition for the occurrence of thermohaline mixing is given by

$\frac{\varphi}{\delta} \nabla \mu \leq \nabla-\nabla_{\mathrm{ad}} \leq 0$,

where $\varphi=(\partial \ln \rho / \partial \ln \mu)_{P, T}, \delta=(\partial \ln \rho / \partial \ln T)_{P, \mu}, \nabla_{\text {ad }}$ the adiabatic gradient, and $\nabla=\mathrm{d} \ln T / \mathrm{d} \ln P$. Mixing is treated as a diffusive process and the associated coefficient follows the Kippenhahn et al. (1980) formulation

$D_{t}=C_{t} K_{T}\left(\frac{\varphi}{\delta}\right) \frac{-\nabla \mu}{\nabla_{\mathrm{ad}}-\nabla}$,

where the thermal diffusivity $K_{T}=4 a c T^{3} / 3 \kappa \rho^{2} c_{P}$ and $C_{t}=$ $8 \pi^{2} \alpha^{2} / 3$ is a form factor that depends on the ratio $\alpha=l / d$ of the length $l$ and diameter $d$ of the unstable fluid element (Ulrich 1972).

Following Charbonnel \& Zahn (2007), we assume by default that $C_{t}=1000$. The arguments supporting this high value arise from the necessity to increase the mixing efficiency along the RGB to reproduce the observed decrease in ${ }^{3} \mathrm{He}$ at the luminosity bump. A high value of $C_{t}$ is also in closer agreement with laboratory experiments that show the development of elongated fingers (Krishnamurti 2003). This phenomenological approach remains nevertheless largely uncertain since laboratory conditions are far from being comparable to those prevailing in stellar interiors and the effects of varying $C_{t}$ will be analyzed in Sect. 4 .

\section{Effects of thermohaline mixing}

The effects of thermohaline mixing are investigated by the detailed analysis of a representative SAGB model of initially $9.5 M_{\odot}$ and metallicity $Z=0.02$.

\subsection{Evolution prior to $C$-ignition}

Prior to carbon ignition, thermohaline mixing has almost no effect on the evolution and structure of massive intermediatemass stars. This is caused mainly, in contrast to low-mass stars, by $\mathrm{H}$ burning being powered mostly by the $\mathrm{CNO}$ cycle. The ${ }^{3} \mathrm{He}\left({ }^{3} \mathrm{He}, 2 \mathrm{p}\right){ }^{4} \mathrm{He}$ reactions responsible for producing a mean molecular weight inversion are only active in the uppermost layers of these stars. The thermohaline instabilities that may develop in these remote shells are active during a short period of time and operate to homogenise the surface radiative layers. Deeper inside the star, because of the higher temperatures, most of the ${ }^{3} \mathrm{He}$ initially present and produced by deuterium burning during the pre-main sequence evolution is destroyed efficiently. Therefore, when the first dredge-up occurs after central $\mathrm{H}$ exhaustion, the surface ${ }^{3} \mathrm{He}$ abundance first increases, corresponding to the engulfment of the surviving external ${ }^{3} \mathrm{He}$ pocket, and

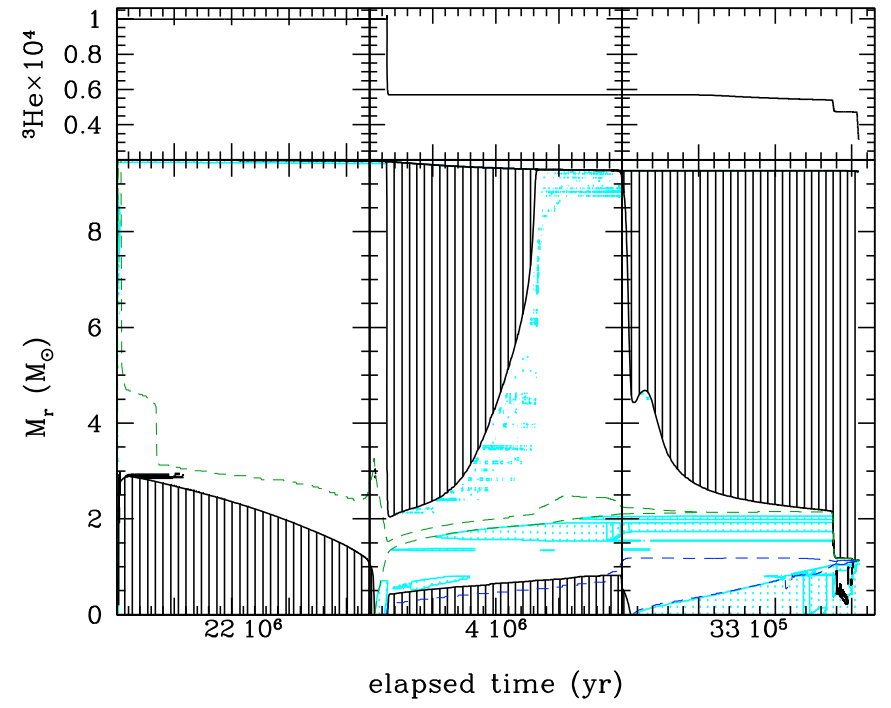

Fig. 1. Top panel: evolution of the surface ${ }^{3} \mathrm{He}$ abundance. Bottom panel: Kippenhahn diagram showing the evolution of the internal structure of a $9.5 M_{\odot}, Z=0.02$ model with thermohaline mixing before carbon ignition. The solid (black) and dotted (cyan) hatched areas correspond to regions unstable with respect to convection and thermohaline mixing, respectively. Note that $\nabla<0$ does not necessarily imply efficient mixing. The $\mathrm{H}$ and He burning shells are also delineated by the green (outer) and blue (inner) dashed lines, respectively.

then decreases as the convective envelope moves inward into the ${ }^{3} \mathrm{He}$-free layers as illustrated at the top of Fig. 2.

At the deepest extent of the convective envelope, the ${ }^{3} \mathrm{He}$ mass fraction has decreased by of factor of two with respect to its initial value, the ${ }^{12} \mathrm{C} /{ }^{13} \mathrm{C}$ ratio has dropped down to $\sim 20$, and the surface ${ }^{14} \mathrm{~N}$ and ${ }^{13} \mathrm{C}$ mass fractions have increased by $\sim 0.45 \mathrm{dex}$ as in the standard case without mixing ${ }^{1}$. On the ascent of the RGB and in contrast to low-mass stars, the $\mathrm{H}$ burning shell (HBS) never crosses the chemical discontinuity left by the envelope during the first dredge-up before He ignites at the center (Fig. 1). The HBS thus always remains detached from the envelope, and the surface abundances are unmodified, at least until the second dredge-up comes into play, i.e. at the time of carbon burning.

\subsection{Carbon-burning phase}

In SAGB stars, carbon ignites off-center in an energetic flash driving a convective zone (e.g., Garcia-Berro et al. 1997; Gil-Pons et al. 2005; Siess 2006). After the decay of the instability, carbon reignites deeper in the star releasing less energy and the structure readjusts into a new configuration in which all the energy produced by the ${ }^{12} \mathrm{C}+{ }^{12} \mathrm{C}$ reactions is carried away by neutrinos. When this "balanced-power condition" (Timmes et al. 1994) is achieved, carbon burning proceeds as a deflagration with the burning front moving inward, dragging behind a convective zone. When the flame reaches the center, the core consists of ${ }^{16} \mathrm{O}(55-60 \%)$ and ${ }^{20} \mathrm{Ne}(28-32 \%)$ plus traces of ${ }^{23} \mathrm{Na}$ and ${ }^{24} \mathrm{Mg}$. The presence of thermohaline mixing will alter this standard picture.

The production of mostly ${ }^{20} \mathrm{Ne}$ (but also of ${ }^{23} \mathrm{Na}$ and ${ }^{24} \mathrm{Mg}$ ) above the degenerate $\mathrm{CO}$ core produces a mean molecular weight inversion and sets the conditions for the development

\footnotetext{
1 Hereafter, a standard model designates a stellar model computed without thermohaline mixing.
} 


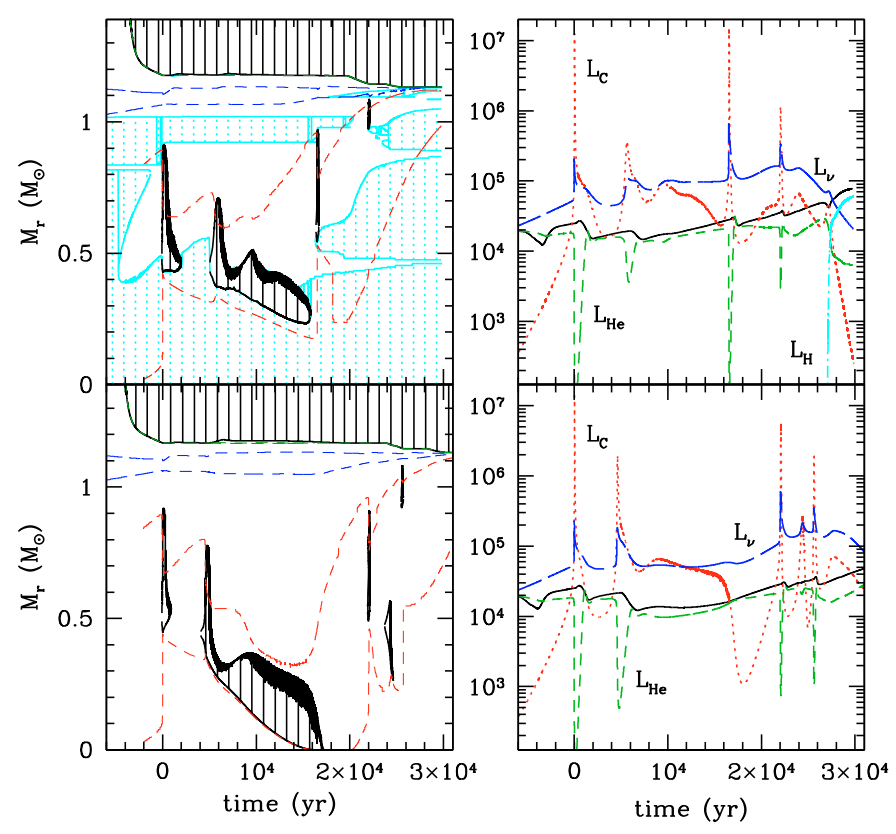

Fig. 2. Compared evolution of a $9.5 M_{\odot}, Z=0.02$ model with (upper panels) and without (bottom panels) thermohaline mixing during the carbon burning phase. The Kippenhahn diagrams are plotted with the same conventions as Fig. 1. The evolution of the various luminosities associated with neutrino losses ( $L_{v}$, blue dotted-dashed line), nuclear energy production by carbon $\left(L_{\mathrm{C}}\right.$ red dotted line), helium $\left(L_{\mathrm{He}}\right.$ green dashed line) and hydrogen ( $L_{\mathrm{H}}$ cyan dashed line if present) burning as well as the surface luminosity (solid black line) are depicted in the right panels for both stars. The origin of time has been reset to the peak carbon luminosity during the carbon flash.

of thermohaline instabilities. When carbon-burning reactions are activated, initially at a low rate close to the center, a $\mu$-inversion develops. Thereafter, as the maximum temperature increases and moves outward, the unstable region (where Eq. (1) is satisfied) grows in mass, and when the flash ignites, it extends throughout the core (Fig. 2). However, until carbon is burnt significantly, $\left|\nabla_{\mu}\right|$ remains small, which prevents efficient mixing being possible (see the diffusion coefficients in Fig. 3). This is why the flash properties are weakly affected by this process. We note however that models accounting for thermohaline diffusion ignite carbon slightly closer to the center in a shell where the degeneracy is a bit lower. As a consequence, the peak carbon luminosity $L_{C}$ as well as the extent of the convective flash are reduced compared to the standard case, but the differences are small $(<10 \%)$. The main differences concern the duration of the convective flash and the time interval between the flash and the flame, both of which are shorter in the standard case.

The most significant impact of this nuclear-induced chemical transport is visible in the evolution of the carbon luminosity. Despite the expansion and cooling of the core after the flash, mixing sustains the nuclear energy production by bringing more fuel into the layers where carbon burning occurs. In Fig. 2 and the top panel of Fig. 6, we clearly see that after the flash extinction, the carbon luminosity reaches its highest value in the models where thermohaline mixing is most efficient. Consequently, when convection resumes, the second peak in $L_{C}$ is much smaller in the thermohaline model since carbon burning operates already at a relatively high rate. This also explains why the temperature is higher than in the standard models (Fig. 4).

The full operation of mixing during the subsequent flame propagation is able to stop the convective flame before it reaches
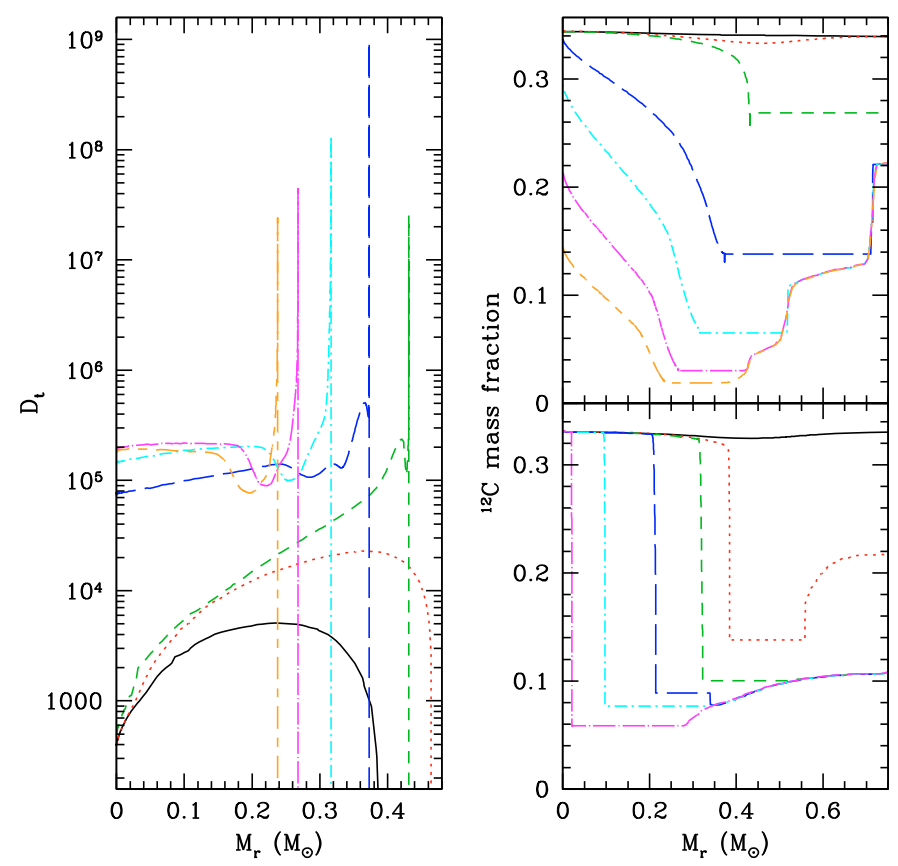

Fig. 3. The left panel depicts the evolution of the thermohaline diffusion coefficient $D_{t}$ at various epochs during the propagation of the flame in our reference $9.5 M_{\odot}, Z=0.02$ model star. The profiles are taken at the following times (in yr as counted in Fig. 2): -4000 (solid), -1000 (dot), 1 (short-dash), 5930 (long-dash), 9500 (dot-short-dash), 12250 (dot-long-dash) and 14250 (short-long-dash). The right panel shows the internal ${ }^{12} \mathrm{C}$ profiles in the standard (bottom right) and thermohaline models (upper right).

the center. To understand how this happens, a view of the internal chemical profiles is particularly enlightening (Fig. 3). In the radiative shells located just below the convective zone where the temperature peaks and the nuclear activity reaches its maximum value ${ }^{2}, \nabla \mu$ is negative. Thermohaline instabilities develop in these layers and allow the transport of chemicals between the convective flame and the CO-rich core. The diffusion coefficient $D_{t}$ is maximal at the edge of the convective boundary mainly because in this region $\nabla \approx \nabla_{\text {ad }}$. Away from the convective front, the transport by radiation and conduction is efficient and, with decreasing temperatures, $D_{t}$ rapidly drops from $10^{7-8} \mathrm{~cm}^{2} \mathrm{~s}^{-1}$ to an almost constant value of $10^{5} \mathrm{~cm}^{2} \mathrm{~s}^{-1}$ (Fig. 3). With these high diffusion coefficients, the carbon present in the inert core is transported outward, through the burning front into the convective flame. As evolution proceeds, the carbon mass fraction in the precursor flame decreases and the nuclear energy production, which depends strongly on the carbon mass fraction $\left(\varepsilon_{\mathrm{C}+\mathrm{C}} \propto \mathrm{X}_{\mathrm{C}}^{2}\right)$ eventually stops. By removing the fuel, thermohaline mixing is thus able to stop the nuclear burning. The resulting impoverishment in ${ }^{12} \mathrm{C}$ also reduces the flame speed, which then provides additional time for further fuel removal. This is in sharp contrast to the standard case where the chemical discontinuity that forms at the edge of the convective flame is maintained at all times during the propagation of the flame to the center.

Another approach to understand the quenching of the flame is to consider the "carbon ignition lines", which delineate the region in the density-temperature plane where the rates of neutrino and nuclear energy production by carbon burning equal each other, i.e. where $\varepsilon_{\mathrm{C}+\mathrm{C}}=\varepsilon_{v}$ (Fig. 4). Because $\varepsilon_{\mathrm{C}+\mathrm{C}}$ also

\footnotetext{
2 This region is traditionally referred to as the precursor flame (Garcia-Berro et al. 1997).
} 


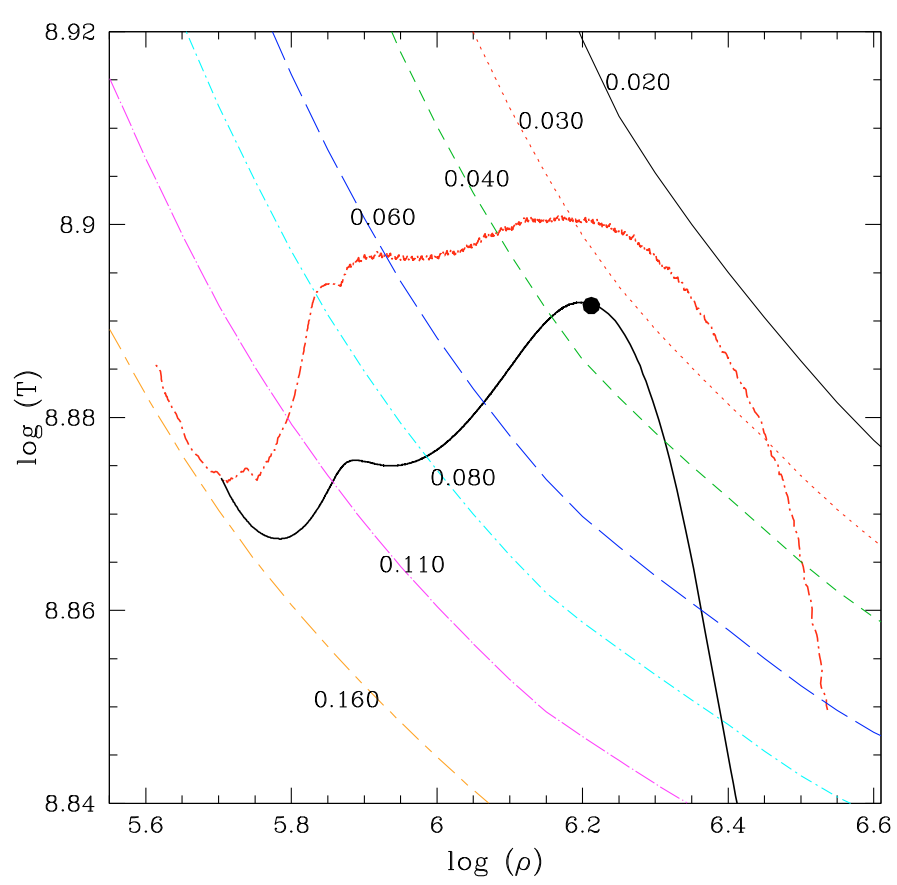

Fig. 4. Carbon ignition lines as a function of the ${ }^{12} \mathrm{C}$ mass fraction. For a given carbon abundance as indicated by the label, the region located below the selected curve is where $\varepsilon_{\mathrm{C}+\mathrm{C}}<\varepsilon_{v}$, i.e. where carbon burning is not self-sustained. The heavy lines represent the evolution during the flame propagation of the temperature and density in the precursor flame (where $\mathrm{T}$ is maximum) in the standard (solid black line) and thermohaline (dotted red line) $9.5 M_{\odot}$ models. The dot on the solid curve refers to the conditions when the flame reaches the center. Note the lower burning front temperature in the standard model.

depends on the carbon mass fraction, a different ignition line exists for each of the ${ }^{12} \mathrm{C}$ abundances. Below each curve, the temperature and density are lower, $\varepsilon_{\mathrm{C}+\mathrm{C}}<\varepsilon_{v}$ and cooling dominates, while above the curve, carbon burning and nuclear heating prevail. It must be emphasized that these ignition lines provide a local criterion for the existence of carbon burning since they were computed using the local properties of the plasma. In a realistic model, the situation is far more complex because the nuclear and neutrino energy production rates are distributed over a large region and energy is transported by both radiation and convection. Therefore, the abundances quoted on the curves do not necessarily correspond to the carbon mass fractions found in our models. Nevertheless, this approach illustrates our point effectively. During the propagation of the flame, mixing lowers the carbon abundance in the burning front and the ignition curves are shifted to regions of higher temperature and density, which eventually becomes out of reach of the flame. Once the ${ }^{12} \mathrm{C}$ abundance is too low, neutrino emission begins to dominate the energy production and the maximum temperature $\left(T_{\max }\right)$ decreases. The quenching of the instability is then irreversible because of the stronger temperature dependence of $\varepsilon_{\mathrm{C}+\mathrm{C}}\left(\propto T^{14}\right)$ compared to that of $\varepsilon_{v}\left(\propto T^{8}\right)$.

Standard models do not have this problem because the carbon abundance in the precursor flame is approximately constant (Fig. 3). Consequently, $T_{\max }$ increases steadily as the burning front moves inward until it reaches the center, where the flame is extinguished because of fuel exhaustion.

It should be noted that the early quenching of the flame is a physical feature that does not depend on the numerical resolution. We completed a series of tests in which the constraint

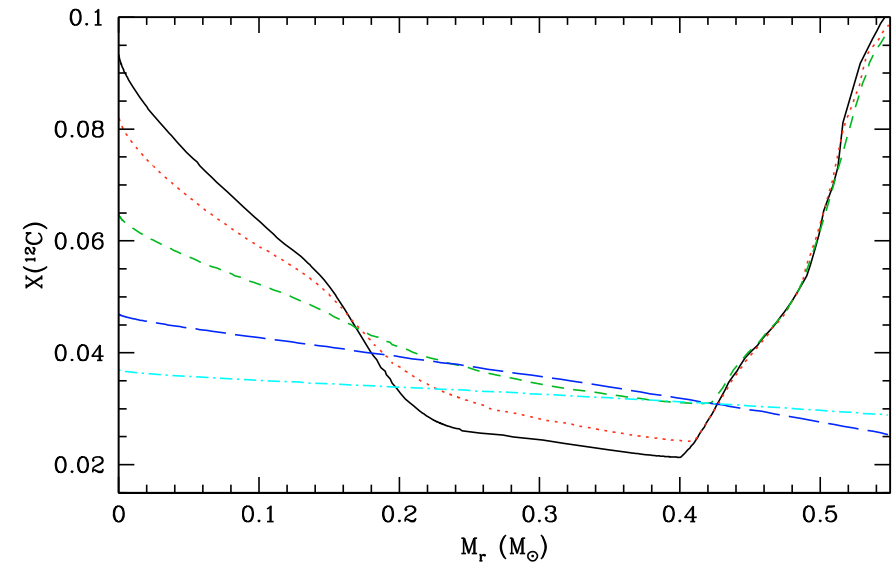

Fig. 5. Evolution of the ${ }^{12} \mathrm{C}$ profiles after the quenching of the convective flame in the $9.5 M_{\odot}$ model.

on the timestep was further increased by imposing a maximum change to the chemical abundances of $2 \%$ between two consecutive models (the reference value is set to be 0.05 ) and the spatial resolution increased, so that the relative variation in the main variables (T, P, $\rho, \mathrm{L}$ ) between 2 mesh-points did not exceed $2 \%$ (the default value being 10\%). These modifications produced timesteps 2 to 3 times shorter than in the standard case and a number of shells ranging between 2000 and 4000. In all cases, the flame was stopped before reaching the stellar center. The differences between these simulations are visible in the duration of the flame or/and on the mass coordinate of the deepest extent of the convection zone, but the overall variations never exceed $\sim 5 \%$. We also report that simulations performed with a lower spatial resolution can result in the occurrence of 3 separate convective episodes, consisting of 2 carbon flashes of decreasing strength and a flame.

\subsection{Evolution of the core composition}

After the quenching of the flame, carbon burning resumes in a shell and initiates the development of secondary convective zones as the peak temperature moves through residual carbon pockets (Fig. 2). However, thermohaline mixing continues to operate in the central regions, smoothing the chemical profiles (Fig. 5). In contrast to the standard case where the central ${ }^{12} \mathrm{C}$ mass fraction drops suddenly when the flame reaches the center, the transport of chemicals produces a continuous decline in the central carbon abundance (Fig. 6) concomitant with a decrease in ${ }^{16} \mathrm{O}$ and an increase in ${ }^{23} \mathrm{Na}$ and ${ }^{20} \mathrm{Ne}$. In the end, the models computed with thermohaline mixing have a higher amount of carbon in their core (Table 1).

During the subsequent thermally pulsing AGB phase, the core mass increases due to mass accretion from the $\mathrm{H}$ and He burning shells. In the course of its evolution, if the core mass reaches the critical value of $1.37 M_{\odot}$ (Nomoto 1984), electron capture (EC) reactions are activated and trigger the hydrodynamical collapse of the core. The initial mass of single stars ending their life as EC-supernovae (EC-SN) is highly uncertain and depends on the mixing processes operating during central $\mathrm{H}$ and He burning and on the competition between core growth and mass loss rates during the TP-SAGB phase. The studies of Siess (2007) and Poelarends et al. (2008) indicate that the mass range of EC-SN progenitors is probably small $\left(<1 M_{\odot}\right)$, supporting the idea of a binary scenario in which an oxygen-neon $(\mathrm{ONe})$ white 


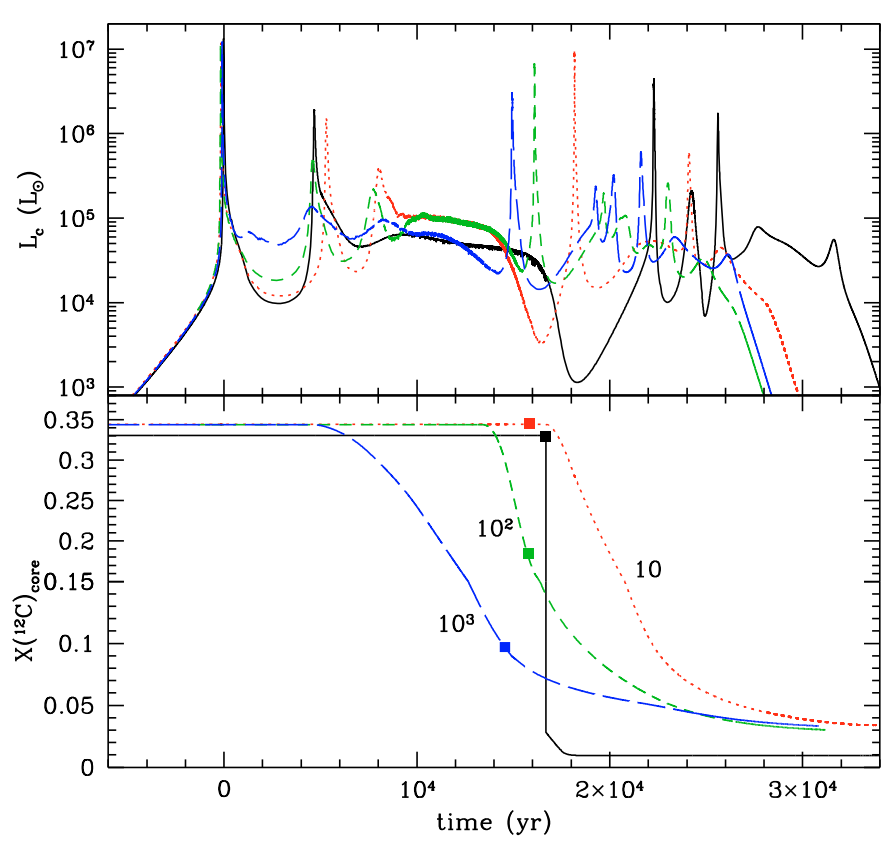

Fig. 6. top panel: evolution of the carbon luminosity. The solid line corresponds to the standard case while the dotted short- and long-dashed lines to the mixing case with $C_{t}=10,10^{2}$ and $10^{3}$, respectively. lower panel: Evolution of the central ${ }^{12} \mathrm{C}$ mass fraction for the same stars. Dots on the curves indicate the time of extinction of the convective flame.

dwarf accretes material from a companion star (Podsiadlowski et al. 2004). However, most importantly for our purpose, the details of the explosion depend sensitively on the core composition. Gutiérrez et al. (2005) showed that traces of unburnt carbon as low as $1.5 \%$ in mass can trigger a low- to moderate-density explosion that would completely disrupt the star instead of a high density explosion where the star collapses to form a neutron star. Our results including thermohaline mixing indicate that the amount of carbon left in the core is large enough to produce a complete disruption of the $\mathrm{ONe}$ core. Thermohaline mixing may thus alter the fate of the star as well as nucleosynthesis but these aspects should be explored.

\subsection{The thermally pulsing SAGB phase}

We continued the evolution of our reference $9.5 M_{\odot}$ model during the thermally pulsing super-AGB (TP-SAGB) phase. In SAGB stars, the temperature below the convective envelope is too high $\left(>5 \times 10^{7} \mathrm{~K}\right)$ for ${ }^{3} \mathrm{He}$ to survive, and with a mass fraction as small as $10^{-11}$, its nuclear burning is unable to modify significantly the mean molecular weight and thus trigger mixing. The peak flux of the ${ }^{3} \mathrm{He}\left({ }^{3} \mathrm{He}, 2 \mathrm{p}\right){ }^{4} \mathrm{He}$ reaction is located within the convective envelope and, given the short convective turnover timescale, $\nabla \mu$ is unable to increase. Therefore, thermohaline mixing is inhibited during the TP-SAGB phase and this conclusion can be extended to intermediate-mass AGB stars that experience hot bottom burning i.e., stars with $M \gtrsim 3-4 M_{\odot}$ (depending on the metallicity). As for the lower mass AGB stars, the possible activation of thermohaline mixing depends strongly on the amount of ${ }^{3} \mathrm{He}$ remaining in the convective envelope. This abundance depends on the mixing history and, in particular, on the efficiency parameter $C_{t}$ (see also Cantiello et al. 2008). As a conclusion, thermohaline mixing does not affect the surface abundances during the TP-SAGB phase.
Table 1. Selected evolutionary properties of the SAGB models as a function of initial mass $M\left(M_{\odot}\right)$ and mixing efficiency $\left(C_{t}\right)$.

\begin{tabular}{crrrccccc}
\hline \hline$M$ & $C_{t}$ & $r_{f}$ & $M_{f}$ & $\Delta t_{f}$ & ${ }^{12} \mathrm{C}$ & ${ }^{16} \mathrm{O}$ & ${ }^{20} \mathrm{Ne}$ & ${ }^{23} \mathrm{Na}$ \\
\hline 9.0 & $10^{3}$ & 2906 & 0.332 & 7495. & 0.050 & 0.524 & 0.313 & 0.032 \\
9.5 & $10^{3}$ & 2895 & 0.232 & 10662 & 0.044 & 0.552 & 0.285 & 0.043 \\
9.5 & $10^{2}$ & 1465 & 0.053 & 9549 & 0.030 & 0.526 & 0.326 & 0.035 \\
9.5 & 10 & 782 & 0.006 & 8991 & 0.033 & 0.530 & 0.318 & 0.036 \\
9.5 & 0 & 0.0 & 0.0 & 13061. & 0.010 & 0.569 & 0.291 & 0.054 \\
10.0 & $10^{3}$ & 2678 & 0.157 & 10570 & 0.025 & 0.611 & 0.255 & 0.042 \\
10.5 & $10^{3}$ & 2425 & 0.090 & 10680 & 0.023 & 0.592 & 0.266 & 0.048 \\
11.0 & $10^{3}$ & 1843 & 0.024 & 9813 & 0.013 & 0.592 & 0.270 & 0.052
\end{tabular}

The quantities shown are: the radius $\left(R_{f}\right.$ in $\left.\mathrm{km}\right)$ and mass coordinate $\left(M_{f}\right.$ in $\left.M_{\odot}\right)$ where the convective flame stops, the duration of the convective flame $\left(\Delta t_{f}\right.$ in $\left.\mathrm{yr}\right)$ and the final central abundances of ${ }^{12} \mathrm{C},{ }^{16} \mathrm{O},{ }^{20} \mathrm{Ne}$ and ${ }^{23} \mathrm{Na}$.

\section{Efficiency of thermohaline mixing}

The phenomenological description of thermohaline mixing is affected by significant uncertainties, and to assess these effects, we computed new $9.5 M_{\odot}$ models in which the factor $C_{t}$ was arbitrarily set to 10 and $10^{2}$. By changing $C_{t}$, we directly control the transport efficiency because the characteristic mixing timescale scales as $C_{t}^{-1}$. As is clear from the previous section, thermohaline mixing becomes truly important after carbon ignition. In the period following the quenching of the flash, the arrival of fresh ${ }^{12} \mathrm{C}$ in the nuclear, active layers previously occupied by the convective instability boosts the nuclear energy production. With a higher mixing rate, a larger amount of ${ }^{12} \mathrm{C}$ is injected into the burning layers and a higher carbon luminosity can be sustained. We also note that, with increasing $C_{t}$ and faster chemical transport, the departure from the standard $L_{\mathrm{C}}$ curve in Fig. 6 occurs at earlier times.

The sustained nuclear energy production is unable however to prevent core contraction and the temperature from increasing in the central regions. Carbon eventually reignites at a lowermass coordinate, where ${ }^{12} \mathrm{C}$ is more abundant, and a new convective zone develops that subsequently evolves into a flame. In all our models, the presence of thermohaline mixing prevents the convective zone from reaching the center. With decreasing mixing efficiency, the flame is stopped at a later time and thus penetrates deeper into the core. We also note that the temperature in the burning front is higher when the efficiency is higher. This is a natural response from the structure, which is forced to increase its temperature to cope with the decrease in nuclear energy production induced by the migration of ${ }^{12} \mathrm{C}$ away from the burning front. After the flame extinction, the core composition continues to evolve until it reaches an asymptotic limit (Fig. 6). We see that a faster transport characterized by a higher value of $C_{t}$ produces an earlier decrease in the central ${ }^{12} \mathrm{C}$ mass fraction.

Concerning the final central carbon abundance, our simulations indicate that it is relatively independent of the mixing efficiency (Table 1). A closer look at the chemical profiles reveals that after the quenching of the flame, the ${ }^{12} \mathrm{C}$ profile presents a minimum at a mass coordinate $M_{\text {mix }}$, which varies between $0.25-0.48 M_{\odot}$ and increases with $C_{t}$. At $M_{\text {mix }}, \nabla \mu$ becomes positive and thermohaline mixing is thus confined to the inner shells enclosing $M_{\text {mix }}$. The dilution of the unburnt carbon is thus larger in models computed with a higher $C_{t}$ but this effect is compensated for by the fact that the amount of carbon left after the flame extinction is larger in these models. This is why in our models 


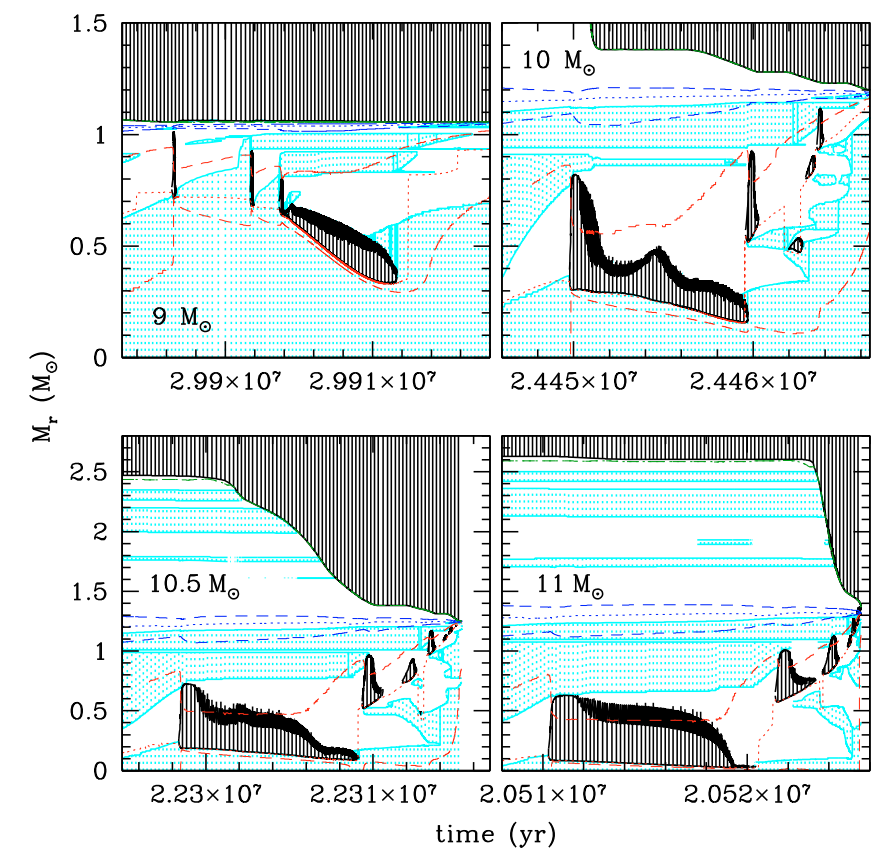

Fig. 7. Kippenhahn diagrams of the carbon burning phase of solar metallicity super-AGB stars in the mass range 9 to $11 M_{\odot}$ with thermohaline mixing. Conventions are the same as in Fig. 1.

the final carbon abundance appears almost independent of the mixing efficiency.

\section{Mixing as a function of initial stellar mass}

The effect of thermohaline mixing in stars of different masses is illustrated in Fig. 7. In all cases, thermohaline mixing prevents the convective flame from reaching the center. In the $9 M_{\odot}$ model, the star experiences 2 short-lived carbon flashes before the convective flame develops. In the higher mass range $\left(M \geq 10 M_{\odot}\right)$, only one convective episode is present, the flash progressively evolving into a flame. The main reason for the absence of a classical flash+flame pattern in these models is due to the fast injection of carbon into the convective burning zone that boosts the nuclear energy production. The extra heat source induced by mixing compensates for the cooling associated with the modest expansion and prevents the drop in both $L_{\mathrm{C}}$ and temperature. As a consequence, carbon burning is maintained at the base of the convective zone and the flash moves inwards instead of receding as in the standard case. This effect is not present in the lower-mass models because carbon ignites at a higher degeneracy. A much stronger flash is generated leading to a more rapid expansion and stronger cooling of the structure that cannot be counterbalanced by the increased nuclear energy production induced by the transport of ${ }^{12} \mathrm{C}$.

The premature quenching of the convective flame leaves unburnt amounts of carbon in the core (Table 1). With increasing initial mass, carbon ignites closer to the center because of the lower degeneracy. It is thus logical that, for a given mixing efficiency, the flame also stops closer to the center and the central carbon mass fraction decreases when the initial mass increases. These computations indicate that, except for the most massive stars $\left(M \gtrsim 11 M_{\odot}\right.$ with our parameters), the central carbon mass fraction remains above the theoretical threshold of 0.015 for core disruption.

\section{Summary}

We have analyzed the effect of thermohaline mixing in the evolution of super-AGB stars in the mass range 9-11 $M_{\odot}$. We have found that this instability operates after the carbon is ignited. The efficient transport of chemicals and particularly of ${ }^{12} \mathrm{C}$ away from the core deprives the burning front of the fuel needed to power the combustion. The nuclear energy production consequently drops and the flame quenches before it reaches the center. This leaves relatively large amounts of unburnt ${ }^{12} \mathrm{C}$ in the central regions $\left(X_{\mathrm{C}} \simeq 2-5 \%\right)$ that may have important consequences if the stellar core is able to reach the critical mass of $\sim 1.37 M_{\odot}$ and activate electron-capture reactions. We also found that the final central ${ }^{12} \mathrm{C}$ abundance does not depend strongly on the mixing efficiency. The core composition of the most massive SAGB stars is less affected by thermohaline mixing because the carbon ignites closer to the center in these models. During the thermally pulsing super-AGB phase, the high temperature encountered at the base of the convective envelope prevents the establishment of a mean molecular inversion because of the absence of ${ }^{3} \mathrm{He}$. Hot bottom burning thus inhibits the thermohaline mixing. To conclude, we emphasize that other hydrodynamical instabilities, associated for example with rotation or magnetic fields, may interfere with thermohaline mixing and erase $\nabla \mu$ before this instability can develop. Multi-D hydrodynamical simulations are required to clarify this issue and provide a clearer description of this process.

Acknowledgements. Marc Joos participated in the early exploration of thermohaline mixing in SAGB stars as part of his bachelor project and is acknowledged for his input. LS is FNRS research scientist. The author also thanks the anonymous referee for constructive comments. This research was supported under Australian Research Council's Discovery Projects funding scheme (project number DP0877317) and by the Communauté française de Belgique - Actions de Recherche Concertées.

\section{References}

Bitzaraki, O. M., Rovithis-Livaniou, H., Tout, C. A., \& van den Heuvel, E. P. J. 2004, A\&A, 416, 262

Cantiello, M., \& Langer, N. 2008, IAU Symp., 252, 103

Charbonnel, C., \& Zahn, J.-P. 2007, A\&A, 467, L15

Eggleton, P. P., Dearborn, D. S. P., \& Lattanzio, J. C. 2006, Science, 314, 1580

Eggleton, P. P., Dearborn, D. S. P., \& Lattanzio, J. C. 2008, ApJ, 677, 581

de Grève, J. P., \& Cugier, K. 1989, A\&A, 211, 356

Garcia-Berro, E., Ritossa, C., \& Iben, I. 1997, ApJ, 485, 765

Gil-Pons, P., \& Garcia-Berro, E. 2002, A\&A, 296, 589

Gil-Pons, P., Suda, T., Fujimoto, M. Y., et al. 2005, ApJ, 433, 1037

Gil-Pons, P., Gutiérrez, J., \& Garcia-Berro, E. 2007, A\&A, 464, 667

Grevesse, N., Noels, A., \& Sauval, A. J. 1996, ASPC, 99, 117

Gutiérrez, J., Canal, R., \& García-Berro, E. 2005, A\&A, 435, 231

Iben, I., Ritossa, C., \& Garcia-Berro, E. 1997, ApJ, 489, 772

Kippenhahn, R., Ruschenplatt, G., \& Thomas, H.-C. 1980, A\&A, 91, 175

Krishnamurti, R. 2003, J. Fluid. Mech., 483, 287

Marks, P. B., \& Sarna, M. J. 1998, MNRAS, 301, 699

Nomoto, K. 1984, ApJ, 277, 791

Podsiadlowski, P., Langer, N., Poelarends, A. J. T., et al. 2004, ApJ, 612, 1044 Poelarends, A. J. T., Herwig, F., Langer, N., \& Heger, A. 2008, ApJ, 675, 614

Ritossa, C., Garcia-Berro, E., \& Iben, I. 1996, ApJ, 460, 489

Ritossa, C., Garcia-Berro, E., \& Iben, I. 1999, ApJ, 515, 381

Siess, L. 2006, A\&A, 448, 717

Siess, L. 2007, A\&A, 476, 893

Siess, L., \& Arnould, M. 2008, A\&A, 489, 395

Stancliffe, R. J., \& Glebbeek, E. 2008, MNRAS, 389, 1828

Timmes, F. X., Woosley, S. E., \& Taam, R. E. 1994, ApJ, 420, 348

Thomas, H.-C. 1967, Z. Astrophys. 67, 420

Ulrich, R. K. 1972, ApJ, 172, 165

Vauclair, S. 2004, ApJ, 605, 874

Wellstein, S., Langer, N., \& Braun, H. 2001, A\&A, 369, 939 